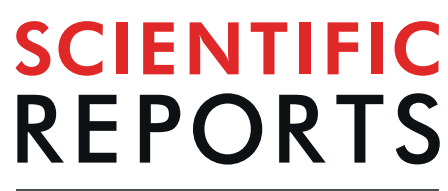

natureresearch

\title{
Chlamydia trachomatis and mycoplasma infections in tubal pregnancy
}

Yang Liu ${ }^{1,6}$, Yunjiang Zhang ${ }^{2,6}$, Dehong Yang ${ }^{3}$, Changjun $\mathrm{Xu}^{4}$, Yajuan Huang ${ }^{4}$, Qing Oing ${ }^{4}$, Daizhu Li ${ }^{4}$, Jing Liao ${ }^{4}$, Yulu Ding ${ }^{4}$, Jiaoyue Zhou ${ }^{4}$, Jie Zhang ${ }^{5}$, Chunyi Sun ${ }^{4 *}$ \& Honglin Zhou ${ }^{4 *}$

Chlamydia trachomatis (CT) infection is an important factor for tubal pregnancy. However, whether Ureaplasma urealyticum (UU) and Mycoplasma hominis (MH) infections are also involved in tubal pregnancy remains unknown. This study is aimed to detect $\mathrm{CT}, \mathrm{UU}$, and $\mathrm{MH}$ in cervical secretions from patients with tubal pregnancy and control women in early pregnancy, to explore their prevalence rates and drug susceptibilities. Analysis was performed on patients with tubal pregnancy and those requiring termination of early pregnancy at $<12$ weeks from July 2013 to March 2014. Cervical secretions were tested for UU/MH with a UU/MH isolation and culture kit and for $\mathrm{CT}$ antigen by an immunochromatographic assay. Mycoplasma samples were tested for resistance to 12 antibiotics. There were no cases of $\mathrm{CT}$ infection detected. Mycoplasma infection rates (single or mixed) were similar in the tubal pregnancy and control groups, but the total rate of infection was higher for tubal pregnancy. All MH samples were sensitive to tetracyclines as well as josamycin and azithromycin. Josamycin and clarithromycin were effective against all UU cultures. Over $50 \%$ of the samples tested were resistant to ciprofloxacin.

The implantation of fertilized eggs outside the uterine cavity is referred to as ectopic pregnancy, with a morbidity of about $2 \%{ }^{1}$. Tubal pregnancy is the most common type of ectopic pregnancy in women of childbearing age, accounting for about $95 \%$ of all cases ${ }^{2}$. Some ectopic pregnancies resolve spontaneously. Because the fallopian tubes are not conducive to placental implantation and embryonic development, the risk of tubal pregnancy rupture and bleeding is enormous ${ }^{3}$. The risk of ectopic pregnancy is increased in women with histories of ectopic pregnancy, infection, infertility, adnexal surgery, appendectomy, and the use of intrauterine devices ${ }^{4}$.

Salpingitis, inflammation of the fallopian tubes, caused by pathogenic microorganisms is a major cause of tubal pregnancy ${ }^{1}$. Previously, bacteria and Neisseria gonorrhoeae were considered the main pathogens in salpingitis $^{5}$. With the progress of medicine and the improvement of detection methods, Chlamydia trachomatis (CT) has been implicated as a leading cause of salpingitis, ectopic pregnancy, and infertility ${ }^{6}$. Ureaplasma urealyticum (UU) and Mycoplasma hominis (MH) are often found alongside $\mathrm{CT}^{7}$. However, the genital tract flora of sexually active healthy women often includes $\mathrm{MH}$ and $\mathrm{UU}^{8}$. Therefore, it is not entirely clear whether $\mathrm{UU}$ and $\mathrm{MH}$ are also implicated in salpingitis or tubal pregnancy ${ }^{9}$. Meanwhile, $\mathrm{MH}$ and UU are implicated in pelvic inflammatory disease, which is related to tubal pregnancy ${ }^{10}$. In addition, both are also involved in adverse pregnancy outcomes $^{11}$. However, a study suggested that there are no associations of $\mathrm{MH}$ and UU serum antibodies with ectopic pregnancy $^{12}$.

Whether treatment for $\mathrm{MH}$ and $\mathrm{UU}$ is required remains controversial, but in some pregnant women, it might be important because co-infection with $\mathrm{UU}$ and $\mathrm{MH}$ could increase the odds of preterm delivery ${ }^{13}$ and low birth weight infants ${ }^{14}$. Antibiotic treatment for MH and UU involves drugs that interfere with protein synthesis and inhibit topoisomerases ${ }^{8}$. However, previous studies have suggested that there is a high degree of antibiotic

${ }^{1}$ Department of Reproductive, The Second Affiliated Hospital of Kunming Medical University, Kunming, Yunnan, 650101, P.R. China. 'Department of Reproductive, The Second People's Hospital of Yunnan Province, Kunming, Yunnan, 650101, P.R. China. ${ }^{3}$ Department of the reproductive gynecology, The First People's Hospital of Yunnan Province, Kunming, Yunnan, 650101, P.R. China. ${ }^{4}$ Department of Gynecology, The Second Affiliated Hospital of Kunming Medical University, Kunming, Yunnan, 650101, P.R. China. ${ }^{5}$ Department of Surgery, The Second Affiliated Hospital of Kunming Medical University, Kunming, Yunnan, 650101, P.R. China. ${ }^{6}$ These authors contributed equally: Yang Liu and Yunjiang Zhang. *email: 787413869@qq.com; km20150515@163.com 


\begin{tabular}{|c|c|c|}
\hline & Tubal pregnancy group $n=81$ & Control group $n=102$ \\
\hline Age, years $($ mean $\pm S D)$ & $29 \pm 2.6$ & $30 \pm 1.8$ \\
\hline Gestational age, weeks (mean \pm SD) & $25.0 \pm 2.8$ & $26.0 \pm 2.1$ \\
\hline \multicolumn{3}{|l|}{ Parity history (n, \%) } \\
\hline 0 & $50(61.7 \%)$ & $31(30.4 \%)$ \\
\hline 1 & $23(28.4 \%)$ & $48(47.1 \%)$ \\
\hline$\geq 2$ & $8(9.9 \%)$ & $23(22.5 \%)$ \\
\hline \multicolumn{3}{|l|}{ History of abortion (n, \%) } \\
\hline 0 & $18(22.2 \%)$ & $42(41.2 \%)$ \\
\hline 1 & $41(50.6 \%)$ & $37(36.3 \%)$ \\
\hline$\geq 2$ & $22(27.2 \%)$ & $23(22.5 \%)$ \\
\hline PID history (n, \%) & 0 & 0 \\
\hline
\end{tabular}

Table 1. Baseline characteristics of the two patient groups. PID = pelvic inflammatory disease.

\begin{tabular}{|l|l|l|l|}
\hline & Tubal pregnancy group $\mathbf{n}=\mathbf{8 1}$ & Control group $\mathbf{n}=\mathbf{1 0 2}$ & P value \\
\hline CT & 0 & 0 & - \\
\hline MH only $(\mathrm{n}, \%)$ & $6(7.4 \%)$ & $2(2.0 \%)$ & 0.141 \\
\hline UU only $(\mathrm{n}, \%)$ & $45(55.6 \%)$ & $46(45.1 \%)$ & 0.160 \\
\hline MH + UU (n, \%) & $3(3.7 \%)$ & $2(2.0 \%)$ & 0.656 \\
\hline Total $(\mathrm{n}, \%)$ & $54(66.7 \%)$ & $50(49.0 \%)$ & 0.017 \\
\hline
\end{tabular}

Table 2. Detection of chlamydia and mycoplasma in cervical secretions. CT $=$ Chlamydia trachomatis; $\mathrm{UU}=$ Ureaplasma urealyticum; $\mathrm{MH}=$ Mycoplasma hominis.

resistance in samples of $\mathrm{MH}$ and $\mathrm{UU}^{8,10,15}$. Therefore, it is important to monitor drug resistance for isolated $\mathrm{UU}$ and $\mathrm{MH}$ samples to ensure that effective treatment is provided to resolve infection, where it is considered appropriate.

The aim of this study was to detect CT, UU, and MH in the cervical secretions of patients with tubal pregnancy and women in early pregnancy, exploring their prevalence rates, drug susceptibilities, and relationships with tubal pregnancy.

\section{Results}

Baseline characteristics. The baseline characteristics of the two groups are shown in Table 1. There were 81 women included in the tubal pregnancy group and 102 in the control group. The mean age and gestational age were similar in both groups.

CT, MH and UU infection rates. No CT infection cases were detected in any of the cervical samples. The rates of $\mathrm{MH}, \mathrm{UU}$, and $\mathrm{MH}+\mathrm{UU}$ infections are shown in Table 2. There were no significant differences between the two groups in terms of $\mathrm{MH}, \mathrm{UU}$, or $\mathrm{MH}+\mathrm{UU}$ infection rates. However, when the overall mycoplasma infection rates were compared, the tubal pregnancy group showed a significantly higher value $(66.7 \% \mathrm{vs} .49 .0 \%, \mathrm{P}=0.017)$.

Antibiotic resistance of the mycoplasma samples. The $\mathrm{MH}$ and $\mathrm{UU}$ samples were tested for resistance to 12 antibiotics, including tetracyclines (tetracycline, doxycycline, and minocycline), macrolides (josamycin, erythromycin, roxithromycin, azithromycin, and clarithromycin), and quinolones (levofloxacin, ciprofloxacin, ofloxacin, and sparfloxacin) as shown in Table 3. The results showed that all MH samples were sensitive to the three tetracyclines tested, and the macrolides josamycin and azithromycin. However, of these drugs only josamycin was effective against all UU samples. The highest level of resistance was found to ciprofloxacin, with over $50 \%$ of all the samples tested being resistant to this drug.

\section{Discussion}

The aim of this study was to detect CT, UU, and MH in the cervical secretions of patients with tubal pregnancy and compare their rates to those of control women in early pregnancy. These findings would provide insights into infection prevalence and drug susceptibilities of the involved infectious agents, and may help establish a potential relationship between infection and tubal pregnancy. As shown above, there were no cases of CT infection, and $\mathrm{UU}, \mathrm{MH}$, and $\mathrm{MH}+\mathrm{UU}$ infection rates were similar in the tubal pregnancy and control groups. However, the total rate of UU and $\mathrm{MH}$ infection was higher in tubal pregnancy. All MH samples were sensitive to tetracyclines as well as josamycin and azithromycin. Josamycin and clarithromycin were effective against all UU cultures. Over $50 \%$ of all the samples tested were resistant to ciprofloxacin.

Many previous studies have shown a link between CT infection and tubal pregnancy ${ }^{16-18}$. However, few studies have investigated the roles of UU and $\mathrm{MH}$. The above results showed no overt associations of UU and $\mathrm{MH}$ infection with tubal pregnancy as the infection rates were similar in both patient groups. This supports the conclusions 


\begin{tabular}{|l|l|l|l|l|l|l|}
\hline \multirow{2}{*}{ Drug resistance } & \multicolumn{3}{|l|}{ Tubal pregnancy group } & \multicolumn{2}{l|}{ Control group } \\
\cline { 2 - 8 } & $\begin{array}{l}\text { MH only } \\
(\mathbf{n = 6})\end{array}$ & $\begin{array}{l}\text { UU only } \\
(\mathbf{n}=\mathbf{4 5})\end{array}$ & $\begin{array}{l}\text { MH+ UU } \\
(\mathbf{n}=\mathbf{3})\end{array}$ & $\begin{array}{l}\text { MH only } \\
(\mathbf{n}=\mathbf{2})\end{array}$ & $\begin{array}{l}\text { UU only } \\
(\mathbf{n = 4 6 )}\end{array}$ & $\begin{array}{l}\text { MH + UU } \\
(\mathbf{n}=\mathbf{2})\end{array}$ \\
\hline Tetracycline & 0 & $3(6.7 \%)$ & $1(33.3 \%)$ & 0 & $4(8.7 \%)$ & $1(50.0 \%)$ \\
\hline Doxycycline & 0 & $1(2.2 \%)$ & 0 & 0 & $1(2.2 \%)$ & 0 \\
\hline Minocycline & 0 & 0 & 0 & 0 & $1(2.2 \%)$ & 0 \\
\hline Josamycin & 0 & 0 & 0 & 0 & 0 & 0 \\
\hline Erythromycin & $1(16.7 \%)$ & $5(11.1 \%)$ & $1(33.3 \%)$ & 0 & $4(8.7 \%)$ & $1(50.0 \%)$ \\
\hline Roxithromycin & $2(33.3 \%)$ & $2(4.4 \%)$ & 0 & $1(50.0 \%)$ & 0 & $1(50.0 \%)$ \\
\hline Azithromycin & 0 & 0 & 0 & 0 & $1(2.2 \%)$ & 0 \\
\hline Clarithromycin & $2(33.3 \%)$ & 0 & $1(33.3 \%)$ & 0 & 0 & $1(50.0 \%)$ \\
\hline Levofloxacin & $1(16.7 \%)$ & $3(6.7 \%)$ & $1(33.3 \%)$ & 0 & $8(17.4 \%)$ & 0 \\
\hline Ciprofloxacin & $4(66.7 \%)$ & $26(57.8 \%)$ & $2(66.7 \%)$ & $2(100.0 \%)$ & $28(60.9 \%)$ & $2(100.0 \%)$ \\
\hline Ofloxacin & 0 & $4(8.9 \%)$ & $1(33.3 \%)$ & $1(50.0 \%)$ & $8(17.4 \%)$ & $1(50.0 \%)$ \\
\hline Sparfloxacin & 0 & $18(40.0 \%)$ & $3(100.0 \%)$ & $1(50.0 \%)$ & $22(47.8 \%)$ & $2(100.0 \%)$ \\
\hline
\end{tabular}

Table 3. Drug susceptibility findings. UU = Ureaplasma urealyticum; $\mathrm{MH}=$ Mycoplasma hominis.

of a previous study ${ }^{12}$. However, when the total rates of UU and MH infections were compared, there was a significant difference between the two groups. This suggests that larger studies are needed to fully investigate whether $\mathrm{UU}$ and $\mathrm{MH}$ infections play a role in tubal pregnancy.

In this study, CT was not detected, but this might be due to the low sensitivity of the applied detection method. Cell culture is a more sensitive and specific method for the diagnosis of reproductive tract CT, but it has high requirements for laboratory equipment and operation and takes a long time to produce results; therefore, it is usually not used as a routine clinical examination and for epidemiological screening, but as a reference standard for other methods. The immunochromatographic method in this study employed a commercial kit that is simple to operate and easily generates immediate results, which is suitable for outpatient examination. Its specificity is $100 \%$, for a sensitivity of $75-85 \%$ based on cell culture methods according to the manufacturer. Nucleic acid amplification diagnosis is available for routine clinical use for CT, UU and $\mathrm{MH}$ infection detection, and has higher sensitivity, but is more expensive and therefore cost-prohibitive in many clinics in China. In addition, the results are also not immediately available. As less expensive polymerase chain reaction (PCR) protocols for CT, $\mathrm{MH}$, and UU detection have been developed, sensitivity in future studies might improve ${ }^{19}$.

There is a global variation in the sensitivities of $U U$ and $M H$ samples to different antibiotics ${ }^{10}$. The sensitivity and susceptibility of mycoplasma to antibiotics are also not static ${ }^{20}$. A Serbian study found doxycycline to be the most effective drug $^{8}$ while a Chinese report suggested that josamycin, doxycycline, and minocycline are all effective $^{15}$. Meanwhile, a German study found clarithromycin and josamycin are the most potent macrolides, although doxycycline was considered the first-choice for the treatment of UU infections and may be used for co-infection with $\mathrm{MH}^{20}$. In Greek women with clinical vaginitis, $\mathrm{MH}$ isolates were $100 \%$ susceptible to tetracycline and doxycycline, and tetracycline and doxycycline were also the most effective drugs for UU although some resistant cases were detected ${ }^{21}$. This study showed similar results in that the susceptibilities of the various mycoplasma infections were different. All three tetracyclines and azithromycin were effective against all MH cultures, and clarithromycin was effective against UU; only josamycin was effective against both UU and MH. The discrepant results of these studies emphasize that it is important for drugs to be selected according to drug susceptibility test data, with rational treatment performed to improve the clinical efficacy and reduce the occurrence of drug resistance.

An important point has to be considered. M. hominis is intrinsically resistant to erythromycin and 14and 15-membered macrolides because of a SNP conferring macrolide resistance in the 23S rRNA gene ${ }^{22-24}$. Surprisingly, in the present study, all M. hominis strains were sensitive to azithromycin and to some 14- and 15-membered macrolides. These results highlight the intrinsic uncertainty of drug susceptibility tests. Unfortunately, the strains were not available for retest or for other tests for all patients. A loss of mutation or novel mutations in $23 \mathrm{~S}$ rRNA conferring sensitivity to erythromycin and azithromycin are possible ${ }^{25}$. Sequencing should be performed in the future. Therefore, these results should not be used as treatment guide for clinicians and will require validation in the future.

This study had some limitations. The sample size was quite small, and a larger study in multiple centers might provide more convincing evidence of a difference in mycoplasma infection between the two groups. The retrospective nature of this study is another limitation; indeed, while we can provide data on infection prevalence, it is difficult to draw any conclusions on the roles of these infections in tubal pregnancy. The testing procedures for infection were not as sensitive as PCR based assays that are currently used in some clinics. This may be the reason for the lack of CT detection in this study. In addition, bacterial load was not precisely controlled in susceptibility tests, which may affect MIC readings. We would have tested for Mycoplasma genitalium, which has demonstrated association with tubal pregnancy. Finally, we did not assess the involvement of host parameters such as immune cell profiles and cytokine production, which deserves further attention.

Overall, no CT infection cases were detected in women with tubal pregnancy or normal early pregnancy. UU, $\mathrm{MH}$, and $\mathrm{UU}+\mathrm{MH}$ infection rates were similar in both groups, but the total rate of UU and MH infection was higher in women with tubal pregnancy. Therefore, further studies are required to establish the associations of UU and $\mathrm{MH}$ infections with tubal pregnancy. $\mathrm{UU}$ and $\mathrm{MH}$ samples showed some resistance to antibiotics, with the 
least effective treatment likely to be ciprofloxacin. However, josamycin was fully effective at preventing the growth of mycoplasma cultures in the present study.

\section{Methods}

Patients. A retrospective study was conducted. Patients admitted to the Gynecologic Outpatient Clinic and Gynecological Inpatient Department of Second Affiliated Hospital of Kunming Medical University from July 2013 to March 2014 were selected, and their cervical secretions were collected for mycoplasma culture and Chlamydia trachomatis detection.

This study was approved by the ethics committee of the above hospital, who waived the need for consent because of the retrospective nature of this study. All methods were performed in accordance with the relevant guidelines.

Patients were selected for inclusion in the tubal pregnancy group based on the following criteria: (1) tubal pregnancy, (2) admission to the Gynecological Inpatient Department during the study period, (3) investigation for CT and mycoplasma infections. The control group included individuals meeting the following criteria: (1) requiring termination of early pregnancy at $<12$ weeks; (2) reason for termination not due to an abnormality with the pregnancy, or health risk to the patient or fetus; (3) investigation for CT and mycoplasma infection; (4) treatment during the study period in the Gynecologic Outpatient Clinic. Exclusion criteria for both groups were: (1) a history of tubal pregnancy; (2) use of any antibiotics in the previous two weeks or use of vaginal irrigation within three days.

$\mathrm{B}$ ultrasound was used as the diagnostic method for intrauterine pregnancy, and postoperative pathological examination was the diagnostic technique for ectopic pregnancy.

Data collection. The baseline data of the patients were collected from the hospital database. This included the patient's age, gestational age, delivery time, history of abortion, history of pelvic inflammatory disease, and history of sexually transmitted diseases.

Collection of cervical specimens. Sterilized cotton swabs were inserted $2-4 \mathrm{~cm}$ into the cervical orifice for sampling cervical secretions. Two senior-technicians analyzed the samples on the same day, in a blinded manner.

Detection of UU and HM. Cervical samples were first grown in liquid cultures in an incubator at $35-37^{\circ} \mathrm{C}$. The samples were investigated for UU and $\mathrm{MH}$ with a UU/MH isolation and culture kit (Zhuhai Diel Bioengineering Co., Ltd, China). The media contained urea and arginine to allow the growth of UU and $\mathrm{MH}$, respectively. In addition, the media changed color when urea and arginine were degraded into an alkaline substance because of the $\mathrm{pH}$ indicator phenolsulfonphthalein. After a 48-hour observation, yellow or orange-yellow color showed the sample was negative for $\mathrm{UU}$ or $\mathrm{MH}$; clear transparent red indicated a positive result. A turbid red sample suggested contamination.

The kit also tested the resistance of UU and MH cultures to 12 antibiotics, including tetracycline, doxycycline, minocycline, josamycin, erythromycin, roxithromycin, azithromycin, clarithromycin, levofloxacin, ciprofloxacin, ofloxacin, and sparfloxacin. The highest drug concentration was in the upper row of wells and the lowest in the bottom row. A well-turning red indicated UU or $\mathrm{MH}$ growth; no color change suggested sensitivity to the drug. When only the lower row turned red, mycoplasma organisms were considered to be moderately sensitive; when both the upper and lower rows turned red, mycoplasma organisms were considered to be drug-resistant.

CT detection. An immunochromatographic assay was used to detect CT antigen with the chlamydia trachomatis test kit (Unipath Ltd., UK). A positive test was reflected by a line appearing in the specimen window.

Statistical analysis. Data are mean \pm standard deviation (SD) or frequency and percentage and were analyzed with SPSS 22.0 (IBM Corp., NY, USA). Chi-squared or Fisher Exact tests were performed for between-group comparison, as appropriate. Two-sided $\mathrm{P}<0.05$ was considered statistically significant.

\section{Data availability}

The data set supporting the results of this report are included in the article.

Received: 28 January 2019; Accepted: 1 October 2019;

Published online: 04 November 2019

\section{References}

1. Marion, L. L. \& Meeks, G. R. Ectopic pregnancy: History, incidence, epidemiology, and risk factors. Clin Obstet Gynecol. 55, 376-386 (2012).

2. Varma, R., Gupta, J. Tubal ectopic pregnancy. BMJ Clin Evid (2012).

3. Hsu, J. Y. et al. Disparities in the management of ectopic pregnancy. Am J Obstet Gynecol. 217, 49 e41-49 e10 (2017).

4. Li, C. et al. Risk factors for ectopic pregnancy: a multi-center case-control study. BMC Pregnancy Childbirth. 15, 187 (2015).

5. Wasserheit, J. N. et al. Microbial causes of proven pelvic inflammatory disease and efficacy of clindamycin and tobramycin. Ann Intern Med. 104, 187-193 (1986).

6. Bebear, C. \& de Barbeyrac, B. Genital Chlamydia trachomatis infections. Clin Microbiol Infect. 15, 4-10 (2009).

7. Guven, M. A., Dilek, U., Pata, O., Dilek, S. \& Ciragil, P. Prevalance of Chlamydia trochomatis, Ureaplasma urealyticum and Mycoplasma hominis infections in the unexplained infertile women. Arch Gynecol Obstet. 276, 219-223 (2007).

8. Skiljevic, D., Mirkov, D. \& Vukicevic, J. Prevalence and antibiotic susceptibility of Mycoplasma hominis and Ureaplasma urealyticum in genital samples collected over 6 years at a Serbian university hospital. Indian J Dermatol Venereol Leprol. 82, 37-41 (2016).

9. Taylor-Robinson, D., Jensen, J. S., Svenstrup, H. \& Stacey, C. M. Difficulties experienced in defining the microbial cause of pelvic inflammatory disease. Int J STD AIDS. 23, 18-24 (2012). 
10. Leli, C. et al. Prevalence and antimicrobial susceptibility of Ureaplasma urealyticum and Mycoplasma hominis in a population of Italian and immigrant outpatients. Infez Med. 20, 82-87 (2012).

11. Capoccia, R., Greub, G. \& Baud, D. Ureaplasma urealyticum, Mycoplasma hominis and adverse pregnancy outcomes. Curr Opin Infect Dis. 26, 231-240 (2013).

12. Karaer, A., Mert, I., Cavkaytar, S. \& Batioglu, S. Serological investigation of the role of selected sexually transmitted infections in the aetiology of ectopic pregnancy. Eur J Contracept Reprod Health Care. 18, 68-74 (2013).

13. Lee, M. Y., Kim, M. H., Lee, W. I., Kang, S. Y. \& Jeon, Y. L. Prevalence and Antibiotic Susceptibility of Mycoplasma hominis and Ureaplasma urealyticum in Pregnant Women. Yonsei Med J. 57, 1271-1275 (2016).

14. Bayraktar, M. R., Ozerol, I. H., Gucluer, N. \& Celik, O. Prevalence and antibiotic susceptibility of Mycoplasma hominis and Ureaplasma urealyticum in pregnant women. Int J Infect Dis. 14, e90-95 (2010).

15. Zeng, X. Y., Xin, N., Tong, X. N., Wang, J. Y. \& Liu, Z. W. Prevalence and antibiotic susceptibility of Ureaplasma urealyticum and Mycoplasma hominis in Xian, China. Eur J Clin Microbiol Infect Dis. 35, 1941-1947 (2016).

16. Bakken, I. J., Skjeldestad, F. E. \& Nordbo, S. A. Chlamydia trachomatis infections increase the risk for ectopic pregnancy: a population-based, nested case-control study. Sex Transm Dis. 34, 166-169 (2007).

17. Hillis, S. D., Owens, L. M., Marchbanks, P. A., Amsterdam, L. F. \& Mac Kenzie, W. R. Recurrent chlamydial infections increase the risks of hospitalization for ectopic pregnancy and pelvic inflammatory disease. Am J Obstet Gynecol. 176, 103-107 (1997).

18. Rekart, M. L. et al. Chlamydia public health programs and the epidemiology of pelvic inflammatory disease and ectopic pregnancy. J Infect Dis. 207, 30-38 (2013).

19. Aguilera-Arreola, M. G., Gonzalez-Cardel, A. M., Tenorio, A. M., Curiel-Quesada, E. \& Castro-Escarpulli, G. Highly specific and efficient primers for in-house multiplex PCR detection of Chlamydia trachomatis, Neisseria gonorrhoeae, Mycoplasma hominis and Ureaplasma urealyticum. BMC Res Notes. 7, 433 (2014).

20. Krausse, R. \& Schubert, S. In-vitro activities of tetracyclines, macrolides, fluoroquinolones and clindamycin against Mycoplasma hominis and Ureaplasma ssp. isolated in Germany over 20 years. Clin Microbiol Infect. 16, 1649-1655 (2010).

21. Kechagia, N., Bersimis, S. \& Chatzipanagiotou, S. Incidence and antimicrobial susceptibilities of genital mycoplasmas in outpatient women with clinical vaginitis in Athens, Greece. J Antimicrob Chemother. 62, 122-125 (2008).

22. Bebear, C. M. \& Kempf, I. Antimicrobial therapy and antimicrobial resistance. In: Blanchard A, Browning G.F., editors. Mycoplasmas: Molecular Biology, Pathogenicity and Strategies for Control. Norfolk: Horizon Biosciences (2005).

23. Pereyre, S. et al. Mutations in 23S rRNA account for intrinsic resistance to macrolides in Mycoplasma hominis and Mycoplasma fermentans and for acquired resistance to macrolides in M. hominis. Antimicrob Agents Chemother. 46, 3142-3150 (2002).

24. Valentine-King, M. A. \& Brown, M. B. Antibacterial Resistance in Ureaplasma Species and Mycoplasma hominis Isolates from Urine Cultures in College-Aged Females. Antimicrob Agents Chemother. 61 (2017).

25. Pereyre, S., Renaudin, H., Charron, A., Bebear, C. \& Bebear, C. M. Emergence of a 23 S rRNA mutation in Mycoplasma hominis associated with a loss of the intrinsic resistance to erythromycin and azithromycin. J Antimicrob Chemother. 57, 753-756 (2006).

\section{Acknowledgements}

This work supported by the National Nature Fund (grant number 81860515), the Health Science and Technology Plan Project of Yunnan (grant numbers 2014NS092, 2016NS286, 2016NS287 and 2017NS277), the Yunnan Health Training Project of High Level Talents (grant number H-201629), and the Applied Basic Research Joint Special Fund Project of Yunnan Provincial Science and Technology Department-Kunming Medical University (The mechanism of miR-214/Sema4D in angiogenesis of ovarian carcinoma) (2018FE001-055).

\section{Author contributions}

Yang Liu conceived and supervised the study. Yunjiang Zhang and Jie Zhang designed the experiments. Dehong Yang, Jiaoyue Zhou, and Jing Liao performed the experiments. Yajuan Huang provided new tools and reagents. Daizhu Li developed the new software and performed simulation studies. Yuxia Ding and Changjun Xu analyzed the data. Qing Qing, Yang Liu, and Honglin Zhou wrote the manuscript. Yajuan Huang and Chunyi Sun performed manuscript revisions. All authors reviewed the results and approved the final version of the manuscript.

\section{Competing interests}

The authors declare no competing interests.

\section{Additional information}

Correspondence and requests for materials should be addressed to C.S. or H.Z.

Reprints and permissions information is available at www.nature.com/reprints.

Publisher's note Springer Nature remains neutral with regard to jurisdictional claims in published maps and institutional affiliations.

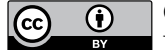

Open Access This article is licensed under a Creative Commons Attribution 4.0 International

License, which permits use, sharing, adaptation, distribution and reproduction in any medium or format, as long as you give appropriate credit to the original author(s) and the source, provide a link to the Creative Commons license, and indicate if changes were made. The images or other third party material in this article are included in the article's Creative Commons license, unless indicated otherwise in a credit line to the material. If material is not included in the article's Creative Commons license and your intended use is not permitted by statutory regulation or exceeds the permitted use, you will need to obtain permission directly from the copyright holder. To view a copy of this license, visit http://creativecommons.org/licenses/by/4.0/.

(c) The Author(s) 2019 